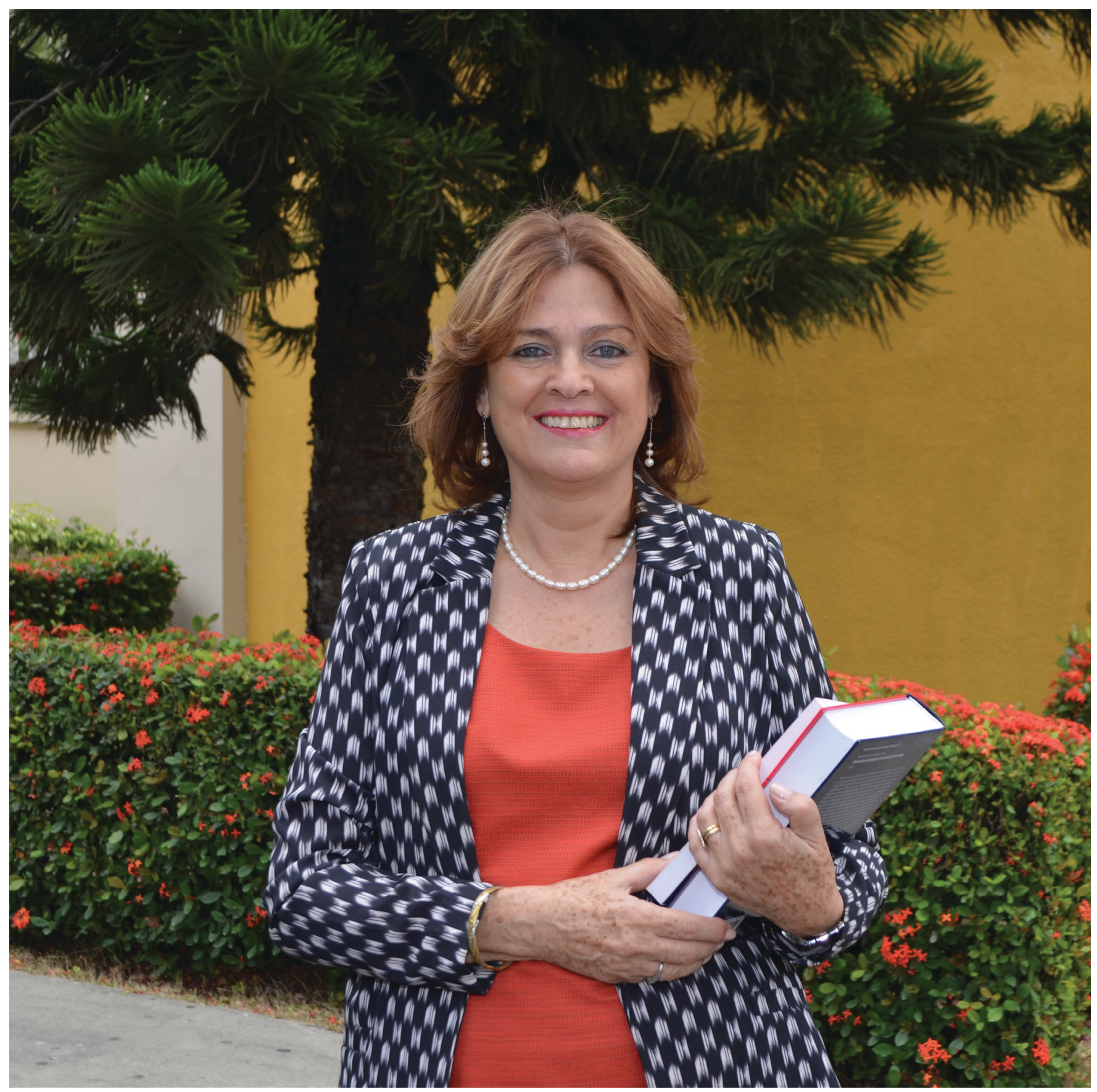

\title{
Entrevista a la profesora Liliana Montenegro
}

\section{Directora del Centro de Excelencia para la Investigación y Difusión de la Lectura y Escritura (CEDILE)}

\begin{abstract}
El Centro de Excelencia para la Investigación y Difusión de la Lectura y Escritura (CEDILE) es una unidad de la Facultad de Ciencias y Humanidades de la Pontificia Universidad Católica Madre y Maestra (PUCMM), cuyo propósito consiste en promover la investigación y la enseñanza de la lengua española como constructora de identidad, como medio para el desarrollo creativo e intelectual de los sujetos y como base para el desarrollo de la criticidad.
\end{abstract}


Entre sus programas formativos, ha desarrollado un Diplomado en Lectura y Escritura a través del Currículo en el nivel Superior. Los resultados de los proyectos desarrollados por los profesores participantes en la primera cohorte de este diplomado fueron presentados en un seminario, llevado a cabo en el campus de Santiago el 21 de marzo de 2014 y el 27 de marzo de 2014 en Santo Domingo. El objetivo esencial de este seminario fue compartir con la comunidad universitaria las experiencias de investigación sobre las prácticas docentes llevadas a cabo por los profesores en sus clases, en las que se aplicaron diversas estrategias de lectura y escritura desarrolladas en el Diplomado, con el fin de lograr un mejor desempeño académico de los estudiantes que cursan las distintas carreras profesionales de grado de la Universidad.

\section{¿Cuándo inició este proyecto?}

LM: Este proyecto se materializó en nuestra Universidad en el año académico 2013-2014, atendiendo a una solicitud de la Vicerrectoría Académica de Grado.

\section{¿Cómo surgió este proyecto?}

LM: El tema de la alfabetización académica consta de antecedentes en la PUCMM que permitieron la sensibilización de profesores y autoridades institucionales sobre la importancia del desarrollo de estrategias de lectura y escritura en las diferentes áreas disciplinares del nivel superior. En efecto, desde el Centro de Desarrollo Profesoral (CDP) de la PUCMM se realizó en el 2009 un sondeo entre el profesorado para indagar sobre las prácticas de lectura y escritura que implementaban en las asignaturas que dictaban en la Universidad. Sus resultados fueron publicados en el Cuaderno de Pedagogía Universitaria (número 12) titulado "Leer y Escribir en la Universidad".

Su propósito fue aportar a la comunidad académica un primer análisis sobre la temática desde la que se podrían impulsar las próximas investigaciones $y$, de alguna manera, propiciar el diseño de políticas de gestión académicas acordes a los datos recogidos. En el diseño, administración del sondeo y en la escritura del artículo participaron profesionales del CDP y de la Universidad, bajo el liderazgo de la Directora del CDP en aquel entonces, Ana Margarita Haché. El cuestionario se administró a 124 profesores del campus Santiago y a 62 del campus Santo Tomás de Aquino.

Los resultados indicaron que los docentes encuestados piensan que "las destrezas de lectura y escritura ocupan un rol tan preponderante como el de construir el conocimiento, organizar el pensamiento, ayudar a la incorporación disciplinar y comunicar conceptos. Sin embargo, los docentes se abstuvieron de reportar el empleo de estrategias didácticas donde el papel del profesorado y del aprendizaje en equipo constituyera una mediación importante para el logro de las expectativas". En palabras de Carlino (2009), parece ser que "se exige escribir, pero no se enseña, los alumnos escriben para demostrar el saber y no para desarrollarlo". Ese desarrollo la mayoría de las veces solo se da "según la posibilidad de recibir orientación de cómo hacerlo".

Otro antecedente importante en nuestra Universidad ha sido los trabajos en el campo de la lectura y escritura que viene realizando el equipo del hoy Centro de Excelencia para la Investigación y para la Difusión de la Lectura y la Escritura (CEDILE), que desde el 2002 lleva a cabo proyectos de formación continua en el área de la

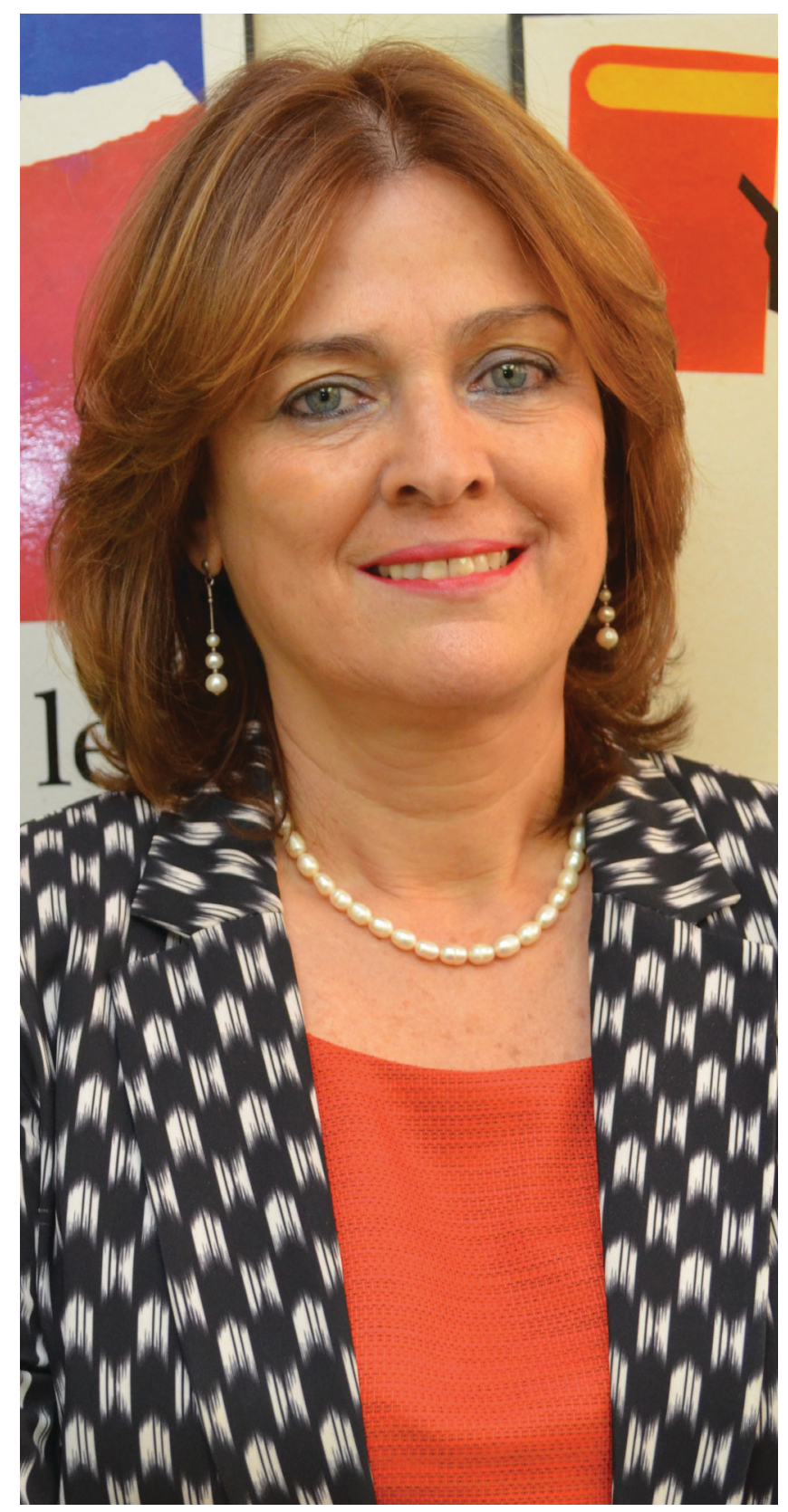


lectura y la escritura a profesores en servicio. Aunque el foco inicial de este trabajo lo constituyeron docentes del nivel primario, el Centro trabajó igualmente estrategias de desarrollo profesoral para los formadores de esos docentes, lo que le ha permitido crear un modelo de capacitación que ha aplicado al programa piloto de lectura y escritura en la Universidad.

El Centro también ha desarrollado seminarios sobre el tema de la lectura y escritura, uno en el año 2010 y otro en el 2012, con motivo del 50 Aniversario de la PUCMM. En este último fue invitada como conferencista la Dra. Paula Carlino, investigadora del Consejo Nacional de Investigaciones Científicas de Argentina y autora de numerosos artículos sobre el tema de la alfabetización académica, publicaciones que han sido inspiradoras para el trabajo desarrollado por el CEDILE.

Por otro lado, la presencia de la Dra. Carlino en el país permitió intercambios con profesores, directores departamentales, decanos y vicerrectores en los campus de Santiago y de Santo Domingo. Estos encuentros apoyaron la compresión de la relevancia del tema de la alfabetizaciónacadémicaparalacomunidaduniversitaria.

¿En qué consistió el trabajo que presentaron en el marco del Seminario Leer y Escribir a través del currículo en el Nivel Superior?

LM: Institucionalmente la Vicerrectoría Académica de Grado trazó una meta en este campo del saber y solicitó a nuestro Centro desarrollar un trabajo piloto.

Luego de múltiples lecturas e indagaciones sobre el tema, reuniones con lingüistas y académicos de la Universidad para compartir el diseño del proyecto, un grupo piloto de 20 profesores de Medicina, Terapia Física, Arquitectura, Matemática, Administración de Empresa, Derecho, Comunicación Social, Ingeniería Industrial, Ingeniería Civil, Ciencias Básicas, Gestión Financiera y Auditoría, Humanidades y Administración Hotelera, de Santiago y de Santo Domingo, cursaron voluntariamente el Diplomado en Lectura y Escritura, durante dos períodos académicos.

Cabe destacar que los profesores participantes en el grupo piloto merecen un reconocimiento especial pues, en medio de su trabajo regular, dedicaron tiempo y energías para realizar una experiencia retadora por la que, según sus mismas opiniones "ya no son los mismos".

Las opciones teóricas en las que se basó esta experiencia atañen a las distintas cuestiones implicadas en la formación e incluyen marcos conceptuales diferentes, pero convergentes. Los trabajos de Carlino, Pipkin, Reynoso, Nogueira, Padilla, Douglas y López, Natale, Narvaja de Arnoux en Argentina; de Bazerman, en California; Cassany, en España; de Martínez, en Colombia; de Parodi, en Chile y os del equipo interdisciplinario de la Universidad de Puebla, México, constituyeron sólidos puntos de partida para el marco referencial desarrollado por el Diplomado de la PUCMM.

\section{¿Cómo se concibe desde el CEDILE la lectura y la escritura?}

LM: La lectura y la escritura son prácticas sociales que incluyen diversos géneros discursivos y ponen en juego actividades cognitivas por parte de quien las lleva a cabo, pudiendo resultar instrumentos que cambian el trabajo de la mente humana e inciden en la construcción del propio conocimiento (Bazerman, 2009; Scardamalia \& Bereiter, 1985, citados por Carlino, 2010).

Las corrientes que impulsan ocuparse de estas prácticas son el movimiento escribir a través del currículo, vinculado a escribir para aprender y escribir en las disciplinas, que promueven la inclusión de la lectura y la escritura en cada materia porque favorecen el aprendizaje de los contenidos a través de la participación activa de los estudiantes y porque tienen especificidades disciplinares que sólo se pueden aprender situadamente.

\section{¿Cuál fue el enfoque en el Diplomado con respecto al proceso de enseñanza aprendizaje en esta temática?}

LM: Se concibió el aprendizaje desde una concepción constructivista-piagetiana y sociocontructivista Vygostkyano-Wertschiano. Asimismo, en contraposición a las ideas del aprendizaje como recepción pasiva y como una tarea descontextualizada y solidaria, el Diplomado se inscribió en las corrientes del aprendizaje situado, llevando a la práctica las concepciones de comunidad de práctica, participación y aprendizaje guiado (Artemega, 2008), que ha permitido comprender el reto que conllevan los aprendizajes de los profesores destinatarios de la formación en servicio y los aprendizajes de sus estudiantes universitarios.

Por otro lado se ha tenido en cuenta que la afectividad, la construcción de la autoimagen, el vínculo con los pares y con la autoridad juegan un papel importante en el aprendizaje. En efecto, las actitudes emocionales asumidas por los profesores y los estudiantes conllevan consecuencias que pueden afectar el proceso de enseñanza aprendizaje. Si la enseñanza potencia el conocimiento y también el aumento de confianza en sí mismos de los estudiantes y su entusiasmo por aprender individual y colectivamente, los resultados serán más fructíferos y podrán sostenerse en el tiempo. Sobre la formación de docentes en servicio y siguiendo a Nemirovsky $(1992,1997)$, se postuló que se trata de una 


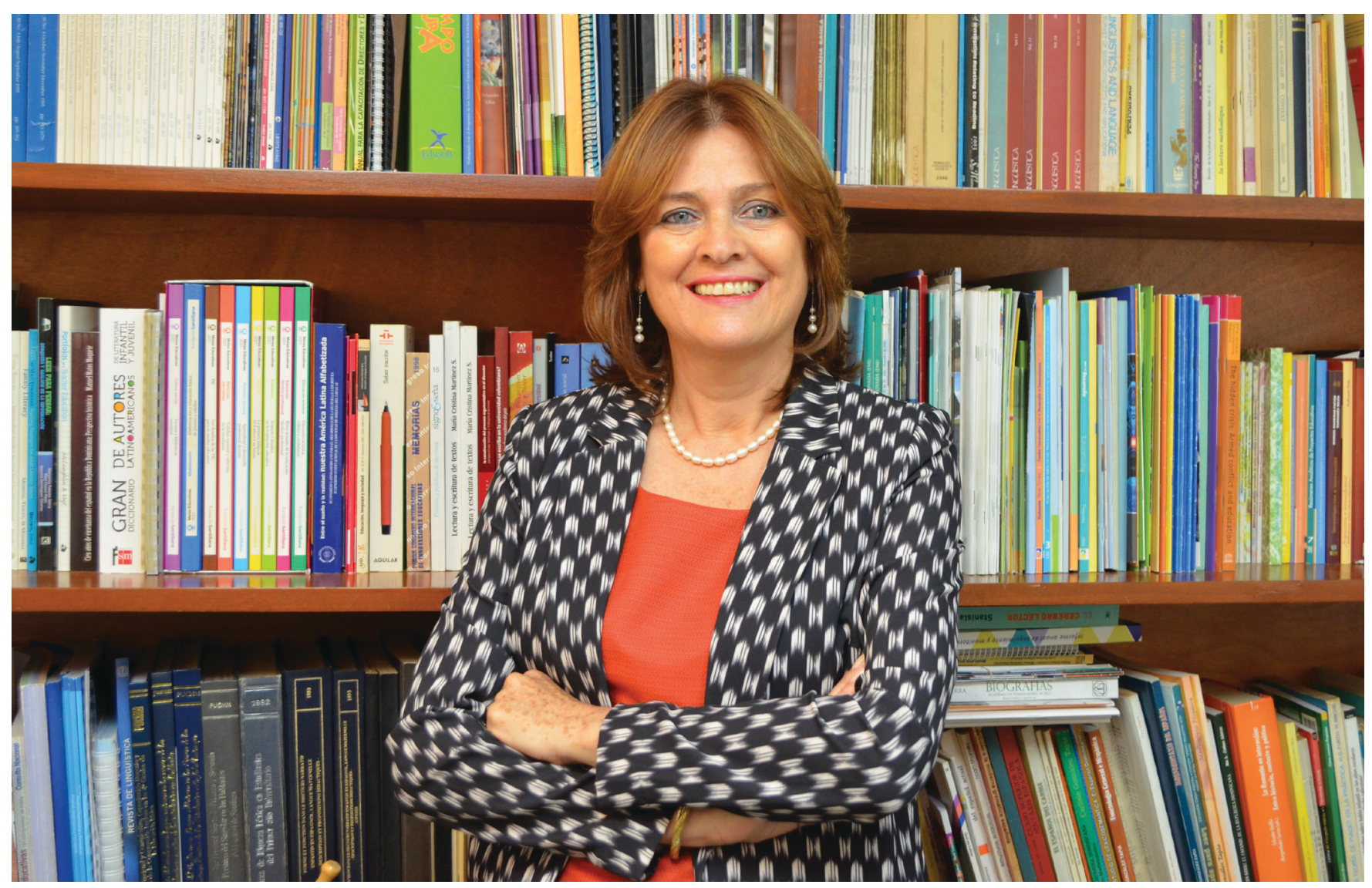

experiencia vital, en la cual están involucrados procesos cognitivos y también cambios en la subjetividad del docente vinculados a su lugar en la sociedad y a su relación con los otros.

El Diplomado planificó, puso en funcionamiento y analizó situaciones didácticas de lectura y escritura en las disciplinas para favorecer la reflexión sobre la práctica en aras de mejorarla. El haber contado con profesores acompañantes en el aula, 13 entre Santiago y Santo Domingo, que horizontalmente interactuaron con los participantes, constituyó parte del éxito de la experiencia.

Por último, destaco que encaramos este trabajo como una tarea investigativa sobre la práctica docente (Hutchings, 2000; Schulamn, 2011) o una investigación en la acción (Altrithcher et al, 2002). En ambos casos, los problemas que se encuentran al enseñar y formar al profesorado, de ser algo indeseable a evitar, devienen el punto de partida para que los docentes y los formadores de docentes continuemos aprendiendo de nuestra práctica, pensándola no sólo en cumplir metas, sino en ir entendiendo por qué ciertas propuestas funcionan o no en determinados contextos y cómo crear otras que puedan ajustarse a las metas y, a la vez, a los contextos en los que trabajamos.

De esta forma, la enseñanza se documenta para dejar de ser una actividad privada del docente o formador y se convierte en una propiedad colectiva, sujeta a difusión, a crítica y al enriquecimiento por parte de la comunidad académica.

Los trabajos que se presentaron en el seminario, desde sus actores, profesores provenientes de las Facultades de Ciencias y Humanidades, de Ciencias Sociales y Administrativas, de Ciencias de la Salud y de Ciencias de la Ingeniería de la PUCMM, se inscriben en esta línea de investigación sobre la práctica docente. Los proyectos que estos profesores llevaron a cabo no se realizaron en el marco de la investigación tradicional, sino que persiguen buscar respuestas a problemáticas que se les han presentado en el contexto de sus aulas, con su grupo de estudiantes. Allí pusieron en práctica estrategias de lectura y/o escritura para mejorar el desempeño académico de esos estudiantes. Sus hallazgos y recomendaciones no buscan realizar generalizaciones sino mejorar la calidad académica.

Estos trabajos nos muestran que es posible ocuparnos de la lectura y la escritura en el nivel superior y nos dan esperanza para pensar que las clases universitarias pueden ser diferentes, propiciando el aprendizaje activo de los estudiantes y el desarrollo del pensamiento analítico y crítico, como una poderosa herramienta para que se conviertan en miembros activos de las disciplinas que decidieron estudiar. 